\title{
Unified Debugging of Distributed Systems with Recon
}

\author{
Kyu Hyung Lee Nick Sumner Xiangyu Zhang Patrick Eugster \\ Department of Computer Science, Purdue University, West Lafayette, IN 47907, USA \\ $\{$ kyuhlee, wnsumner, xyzhang, p\}ecs.purdue.edu
}

\begin{abstract}
To scale to today's complex distributed software systems, debugging and replaying techniques mostly focus on single facets of software, e.g., local concurrency, distributed messaging, or data representation. This forces developers to tediously combine different technologies such as instructionlevel dynamic tracing, event log analysis, or global state reconstruction to gradually explain non-trivial defects.

This paper proposes Recon, a debugging system that provides iterative and interactive homogeneous debugging services. As related systems, Recon promotes SQL-like queries for debugging distributed systems. Unlike other approaches, however, Recon allows for all system artifacts including nodes, communication channels, events, or instructions to be uniformly described by relations. Also, an application in Recon originally runs with a lightweight logger that only collects replay logs for individual nodes. Developers debug a complete program by replaying the execution with fine-grained instrumentation that is capable of exposing instruction-level information.

We illustrate the effectiveness of Recon on programs as diverse as BerkeleyDB, i3/Chord, RandTree, and Pastry. Our evaluation includes executions in local clusters as well as in Amazon EC2 and exhibits an unreported bug in RandTree.
\end{abstract}

Keywords-Software reliability, distributed systems, debugging, replay, instrumentation

\section{INTRODUCTION}

As computing infrastructures continue to become more powerful and pervasive, software for these systems continues to increase in complexity. As a consequence, debugging and replaying techniques are facing increasing scalability challenges, in particular for distributed systems. A natural response to these challenges is to focus on subsets of the equation, that is, to focus on an individual abstraction such as local concurrency, distributed messaging, or data representation. The downside of this approach is that it fails to capture the oftentimes subtle interactions of the different abstractions. Developers are thus commonly forced to manually combine technologies such as instruction level dynamic tracing, event log analysis, or global state reconstruction to gradually explain non-trivial defects that cross the boundaries of individual abstractions.

This paper proposes Recon, a homogeneous debugging system that provides iterative and interactive debugging services through a unified relational view of pervasive distributed systems. Figure 1 presents an overview of our approach. Originally, the distributed application runs with a

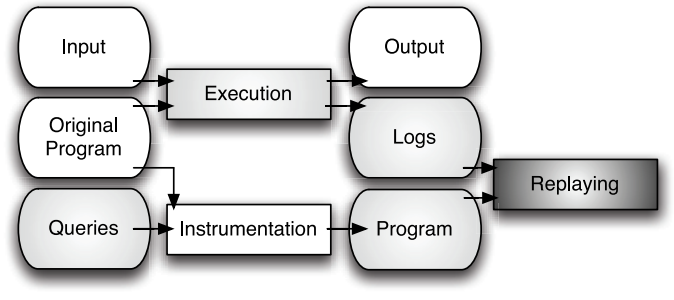

Figure 1: Overview of the Recon approach. The original program executes in a lightweight logger. The replay occurs with heavyweight instrumentation for fine-grained inspection.

lightweight logger that collects minimal logs for deterministic replay of individual nodes. Developers then debug a complete program by replaying the execution with heavyweight instrumentation that is capable of exposing instruction-level information. Like related systems (e.g., [25], [18], [11]), Recon supports SQL-like queries for describing system artifacts that are of interest. Unlike these systems, Recon supports the uniform description by relations of all system artifacts, from the highest level to the lowest level, including nodes, communication channels, messages, event causality and dependencies, method invocations, instructions, and dependencies between instructions; also, Recon does not impose a specific programming language, and supports online analysis as well as replay. Due to resource constraints, most relations are populated in a demand-driven way. In other words, large tables are not explicitly maintained. Rather, queries are compiled into program instrumentation that is executed during replay to answer the query. Developers iteratively refine queries based on previous results. Such a unified view is highly beneficial because the onus is no longer on the developer but on the Recon query system to decide what level of instrumentation to employ to efficiently collect the queried information.

In summary, this paper makes the following contributions:

- Debugging of distributed systems based on unified views. Recon enables a unified view that overcomes the heterogeneous requirements for various technologies in debugging distributed systems. The key idea is to formulate all system artifacts, system wide or node local, event level or instruction level, static or temporal, as relations. Debugging is carried out by writing SQLlike queries over any number of such relations. 
- Compilation of queries to various instrumentation levels. Due to resource constraints, most relations are not explicitly maintained by Recon. We describe how Recon's query compiler translates queries into event log filtering components and code instrumentation. Executing these evaluates queries on demand.

- Separation of lightweight logging and fine-grained replay. Most existing logging and replay techniques are monolithic. That is, they are integrated into one tool that is either lightweight, and hence adequate for production runs but not sufficiently powerful for debugging, or relies on expensive infrastructures such as virtual machines that favor functionality over efficiency. Recon separates logging from replay to avoid expensive instrumentation during original executions. More powerful heavyweight instrumentation is only activated during replay.

- Evaluation of efficiency and effectiveness. We evaluate the efficiency and effectiveness of Reconon a diverse set of distributed programs and their bugs. The results conducted in a local cluster as well as in Amazon Elastic Compute Cloud (EC2) show that our recording overhead is only about $3 \%$ and that instrumented replay causes a constant factor slowdown, depending on the queries. 8 bugs, including a previously unreported bug in RandTree, are effectively resolved by querying various levels of information.

Roadmap. This paper presents Recon in a top-down manner: Section II uses real examples to illustrate the use of Recon. Section III presents a design overview of Recon's architecture. Section IV introduces Recon's debugging view. Section V presents the separation of logging from instrumented replay in Recon. Experimental results are presented in Section VI. Section VII discusses related work. Section VIII concludes with final remarks.

\section{OVERVIEW BY EXAMPLES}

In this section, we present an overview of our technique through examples. The sample bugs are from a Macedon [2] implementation of the Chord [27] distributed hash table protocol. Chord maps a key to a node so that the corresponding data item can be stored and maintained in that node. Chord has been extensively used in pervasive computing environments [4]. The protocol is able to find the host for a key in $O(\log N)$ within $N$ nodes, and to handle nodes joining and leaving at a cost of $O\left(\log ^{2} N\right)$.

The Macedon Chord was found to have a number of bugs in [14]. Some of them cause failures that are local to the node, i.e., they do not manifest directly in the interaction with other nodes. Let us first consider an uninitializedpointer-dereference bug that crashes the local execution. In the beginning, the observable symptom is merely the crash. The developer may have no idea what caused the crash. It may be an incorrect local assignment or faulty

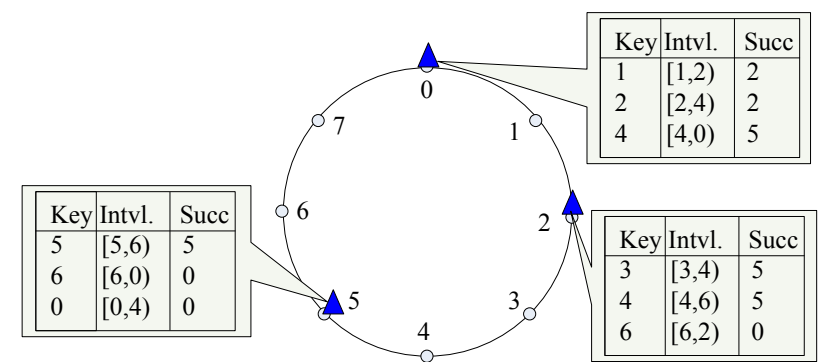

Figure 2: Triangles represent nodes. Each table denotes a partial image of global key distribution. More particularly, a key value is stored in node succ; queries for key values in intvl. should be relayed to node succ.

interaction with other nodes. With our technique, the system is originally running with a lightweight logger. Upon the crash, replay logs are collected from nodes for debugging. The distributed execution is described by a set of conceptual relations that contain information such as values defined or used at a given execution point, data and control dependences of a given statement execution, and node interactions. The developer can write queries about such relations as though the relations were fully populated. In the above crash, suppose the failed node $n$ crashed at execution point $t$ when accessing variable $d$. The simplest way to proceed is to query when $d$ was defined by writing the following query.

\section{SELECT definition FROM Data_Dependence \\ WHERE use $=\mathrm{t}$ AND variable $=\mathrm{d}$ AND host $=\mathrm{n}$}

The query compiler generates instrumentation that collects data dependences. Then the execution of $n$ is replayed with the instrumentation. The query returns an empty table, indicating the pointer was never initialized.

Consider a more subtle Chord-related bug [14]. In the protocol, keys are distributed to nodes and each node has a partial image of the global distribution. In Figure 2, the key domain $[0,7]$ is represented in a ring. Three nodes (triangles) have ids 0,2 , and 5. A key is stored in a node whose id is the key value or the first clockwise node successor of the key value in the ring, e.g., key 6 is stored in node 0 . The tables at each node show the partial images. For example, the first row of the table at node 0 says that it knows keys 1,2 , and 4 are stored at nodes 2, 2, and 5, respectively. Furthermore, keys falling in the range of $[1,2),[2,4)$, and $[4,0)$ are relayed to the nodes specified in the succ column.

In these tables, a pair of (key, succ) is a perfect pair if key=succ. That means the key is stored in the node with the key value as its id. For example, $(5,5)$, denoting that key 5 is stored at node 5, constitutes a perfect pair. According to the Chord protocol, upon the joining of a new node with id $x$, if $x$ falls into the range of (key, succ), indicating the key has a new successor that is closer to being perfect, the succ entry will be updated to $x$, and $x$ becomes the new host of the key. The bug occurs when a pair is perfect; upon the 
joining of any node $x$, the Macedon implementation updates the succ entry to $x$.

In Figure 2, the failure occurs if a new node 6 joins. The table at node 5 is updated by setting the succ of key 5 to 6 due to the fault. Assume the request for key 5 is issued at node 2 . Node 2 relays the request to node 5 according to its table, which returns 6 as the node that hosts key 5 . The attempt from node 2 to access the key at node 6 returns null, which is the failure. The programmer observes the null return at node 2 . A local query performed at node 2 reveals that node 6 was returned as the successor by the previous request sent to node 5 . The programmer now can write another query to retrieve, at node 5, who defined the returned value. The answer to that query reveals that the successor was updated to 6 in the method that handles the joining of new nodes. Note that these queries operate at various levels including communication across nodes. How to answer them is completely handled by our technique and hence transparent to the developer.

\section{DESIGN OVERVIEW}

Recon consists of several components, as shown in Figure 1. During normal execution, a light-weight logger records a minimal application log for deterministic replay, containing non-deterministic effects on the execution such as system calls, CPU instructions, or signals. Recon provides an SQL-based interface to the user for debugging operations; a query compiler generates program instrumentation that is executed during replay.

\section{A. Logging and Replay System}

One unique feature of Recon is the separation of logging and replay to allow for fine-grained replay with necessary heavyweight instrumentation only upon this replay, whilst only requiring lightweight logging at runtime. Most existing logging and replay systems are monolithic, collapsing both the recording and replay capabilities. If low recording overhead is the target, typical logging and replay architectures (e.g. [9]) change the table for system calls (syscalls) or rewriting instructions around syscalls in the executable to redirect them to wrappers. The perturbations imposed on the application execution are usually very limited. During replay, the same interception is performed to read values from the $\log$ file without executing the actual syscalls. Jockey [24] is an example of such a system. Jockey provides lowoverhead logging and deterministic replaying for interactive or distributed programs. Jockey records invocations of nondeterministic system calls and CPU instructions for deterministic replay.

Alas, such OS-level interception is not able to expose fine-grained execution information such as values and dependences of individual instructions necessary for debugging distributed systems. The necessary information is obtained with heavyweight instruction-level instrumentation through dynamic binary instrumentation tools like Pin [17] or Valgrind [20], but at a high cost in terms of runtime overhead. For example, [21] shows the overhead of Pin is up to $60 \%$ for intercepting syscalls. An alternative is to realize the logging and replay functionality in a full-fledged virtual machine that allows intercepting both fine-grained instruction execution and coarse-grained system calls. However, this requires that applications run on the virtual machine.

Recon, in short, uses the Jockey recording/replaying tool as a logger at recording time, yet we implemented a replaying tool which is combined with a dynamic instrumentation tool in Pin. Our replay tools also provide guarantees on deterministic replay of individual nodes by trapping system calls and CPU instructions of application and retrieving events from the log file without actually passing syscall requests on to the OS. We will discuss our implementation in Section $\mathrm{V}$ in detail.

\section{B. User Interface}

Recon provides a declarative interface based on SQL to the developer. Various levels of system artifacts are encoded uniformly into relations. Debugging operations can be composed as queries on one or more such relations. For example, if the developer is concerned about the local instruction-level status and global network status, he/she can easily write a join query of a local table and a global table on conditions about time, etc. Relations are solely conceptual, i.e., they are not explicitly populated and stored. Instead the query compiler translates queries into binary instrumentation at selected program points to evaluate queries online during replay. Note that queries can vary between replays, thus supporting iterative debugging. In the past, SQL interfaces have been proposed for logging and profiling related applications, such as in [25], [16], [11], [18]. Existing work mostly focuses on querying event logs. In other words, an event $\log$ is collected beforehand and serves as a database; events are often at a coarse level, otherwise the space consumption would be prohibitive. Some [11], [18] feature online query evaluation. However, they do not support replay and their schema designs are not debugging oriented. For example, they do not support data and control dependence. In contrast, under the hood of a SQL interface, we support iterative and interactive debugging through replay and expose critical execution artifacts through various relations.

The query language supports standard relational operations including selection, projection, join, anti join, and aggregation, i.e., the GROUPBY operation. The aggregation operation can be useful in finding anomalous behavior. The semantics of the query language also largely follow the standard SQL semantics. The precise information that queries may work with and how the queries can be effectively answered are discussed in the following sections. 


\begin{tabular}{|l|l|l|}
\hline \multicolumn{3}{|c|}{ State (ST) } \\
\hline Field & Type & Description \\
\hline \hline host & INT & host id \\
\hline location & EXE_PNT & exec. point \\
\hline variable & STRING & variable \\
\hline value & BYTE [ ] & untyped value \\
\hline
\end{tabular}

\begin{tabular}{|l|l|l|}
\hline \multicolumn{3}{|c|}{ Output (OUT) } \\
\hline Field & Type & Description \\
\hline \hline host & INT & host id \\
\hline location & EXE_PNT & output point \\
\hline value & BYTE [ ] & output value \\
\hline
\end{tabular}

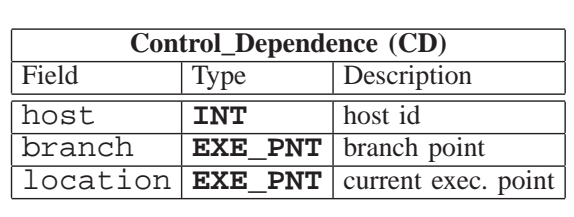

\begin{tabular}{|l|l|l|}
\hline \multicolumn{3}{|c|}{ Control_Flow (CF) } \\
\hline Field & Type & Description \\
\hline host & INT & host id \\
\hline location & EXE_PNT & instruction point \\
\hline pc & INT & program counter \\
\hline instance & INT & instance \\
\hline file & STRING & source file \\
\hline line & INT & line number \\
\hline
\end{tabular}

\begin{tabular}{|l|l|l|}
\hline \multicolumn{3}{|c|}{ Data_Dependence (DD) } \\
\hline Field & Type & Description \\
\hline host & INT & host id \\
\hline definition & EXE_PNT & definition point \\
\hline use & EXE_PNT & use point \\
\hline variable & STRING & variable of dependence \\
\hline
\end{tabular}

\begin{tabular}{|l|l|l|}
\hline \multicolumn{3}{|c|}{ Communication (COM) } \\
\hline Field & Type & Description \\
\hline \hline sender & INT & sender id \\
\hline send_point & EXE_PNT & send point \\
\hline receiver & INT & receiver id \\
\hline recv_point & EXE_PNT & receive point \\
\hline message & BYTE [ ] & message \\
\hline
\end{tabular}

Figure 3: Schema for system properties that may be queried.

\section{SQL INTERFACE}

Recon's logging system collects minimal information for deterministic replay at runtime. Queries are evaluated by executing corresponding instrumentation generated by the query compiler at replay. This section describes schemas, instrumentation generation, and our query compiler.

\section{A. Schemas}

A set of relations that describe common aspects of distributed systems is predefined. These relations include State, Data_Dependence, Control_Dependence, Control_Flow, Output, and Communication. The detailed schemas are shown in Figure 3. In particular, type EXE_PNT is used to describe an execution point. Intuitively, one can interpret it as a triple (source file, line number, instance) that pinpoints a specific instance of a source code statement ${ }^{1}$. In our implementation, we use a more precise representation, which will be discussed in the next section. Variables are represented by their symbolic names.

Relation State records variable information observed within the system. It describes variable values on any host at any point over the duration of the system's execution. Recall that the relation is conceptual and only populated on demand, driven by queries. For example, if a variable value is queried at a specific location $l$ for a node $n$, only the execution of $n$ is replayed, and only the variable value at $l$ is reported. Variables are not necessarily those that are defined or used at a location but rather those that are live.

The Data_Dependence (DD) relation describes which definition (assignment) of a variable is used at a given execution point. For example, executing the code snippet in Fig. 4(a) on node 0 conceptually inserts a tuple (host=0, definition=(f1.c, 1, 1), use=(f1.c, 2, 1), variable=x) in the table, meaning that the use of variable $x$ at the first instance of statement 2 is defined at the first instance of 1 in file $\mathrm{f} 1$. c.

Similarly, Control_Dependence (CD) describes the branch point that directly determines whether or not an

\footnotetext{
${ }^{1}$ A statement can get executed multiple times, leading to many instances.
}

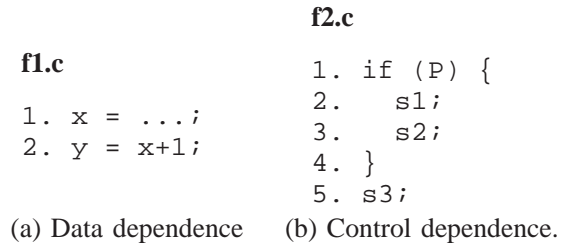

Figure 4: Data $\backslash$ control dependence examples.

instruction at location on host is executed. Assume an execution of the code snippet in Fig. 4(b) takes the true branch and the host is 0. The CD table conceptually contains two tuples (host $=0$, branch $=(\mathrm{f} 2 . \mathrm{c}, 1,1)$, location $=(f 2 . c, 2,1))$ and (host $=0$, branch $=(f 2 . c$, 1, 1), location=(f2.c, 3, 1)). Relation Control_Flow (CF) associates each execution point with a binary program counter, execution instance, and symbolic information.

Besides the above relations describing fine-grained information, we also have relations for coarser-grained information. The output of a host is described by the Output (OUT) relation. The interaction between hosts is captured by the Communication (COM) relation. This relation observes all messages sent between hosts along with the hosts involved and when they sent or received the message in question.

\section{B. Instrumentation Primitives}

We now discuss how we leverage instrumentation to populate the aforementioned relations. The population is controlled by our query compiler.

Representing Execution Point. As discussed earlier, an execution point can be conceptually considered a triple of file name, source code line, and execution instance. However, this is not sufficiently accurate because it is common that a source code line may be composed of multiple statements and even different parts of a statement may lead to different instructions. For example, if a predicate has a conjunctive condition, the different clauses may have different execution instances at runtime. In Recon, we use the instruction count as the internal representation of an execution point (EXE_PNT), i.e., the number of executed instructions up to 
the point in the same process. This is because replay is deterministic (we will discuss how we handle concurrency in Section V-A) and thus the same execution point always has the same instruction count. While instruction count is not a user-friendly representation, the symbolic information can be easily acquired by joining a query with the $\mathbf{C F}$ relation. For example, the following query identifies the source code location that outputs a string "Hello".

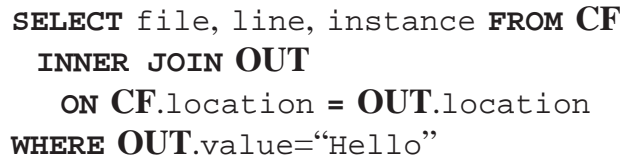

Resolving Variables. For usability, we allow developers to specify variables in their queries using symbolic names. Because the instrumentation to answer queries has to be at the binary level, we have to resolve variables to their addresses. We leverage a tool called dwarfdump [1] to establish the mappings between symbolic names and addresses. For global variables, the tool can directly yield their addresses. The offset of a local variable in the stack frame of its enclosing function can also be identified by the same tool. At runtime - combined with the stack base address which can be acquired from the binary instrumentation tool Pin Recon can compute the stack addresses of local variables. For heap variables, their reference paths are followed to identify their addresses. Note that it must be true that the reference path of a heap variable starts with a global or stack variable, whose address we have already resolved.

Primitives for Relations. To answer a query on the State relation, we first replay the specified host up to the requested execution point; then we resolve the addresses of the specified variables and retrieve their values. For the OUT and COM relations, as both output emission and message sending have to go through system calls, queries can be answered by simple processing of the replay log. Queries of the $\mathbf{C F}$ relation are straightforward to answer as the Pin infrastructure allows identifying the precise PC (program counter) of each instruction and its symbolic information.

The remaining two relations (DD and $\mathbf{C D}$ ) require more effort. Consider the DD relation first. We detect data dependences through a data structure called shadow memory - a small piece of memory allocated inside Pin for each program variable. This memory can be considered the shadow of the respective variable. Upon the definition of the variable, the location that defines the variable is recorded in its shadow memory. Later, when the variable is used, a dependence is detected. Let $S M(x)$ be the shadow memory of $x$. Consider the example in Fig. 4(a). When line 1 is executed, $S M(x)$ is set to 1 . When line 2 is executed, since $x$ is used, a dependence is detected between 2 and $S M(x) \equiv 1$ on variable $x$. We developed an instrumentation module for Pin to carry out the detection.
Control dependences in the CD table are detected as follows. Immediately after a branch point, the linear control flow from that point is dependent upon the branch point because whether or not an instruction is executed is directly determined by the branch. At the postdominator [8] of the branch, i.e, the join point of the branch, execution is no longer determined by the branch point. This implies that control dependence is organized into nested regions much like function calls, and similarly, control dependence can be efficiently observed throughout an execution by pushing a branch point onto a stack called the control dependence stack, and popping once its postdominator is encountered [28]. Thus, in the same way that the function call on the top of the call stack is the currently executed function, the control dependence for the current execution point is observed at the top of the control dependence stack. By maintaining this stack during replay with Pin, control dependences can be detected on the fly. Consider the example in Fig. 4(b). Upon executing the branch point at line 1 , line 1 is pushed onto the control dependence stack. The following executions of lines 2 and 3 are control dependent upon the top entry on the stack, which is line 1 . The entry is popped at the execution of line 5 , the postdominator of 1 , indicating that the following executions are no longer decided by the branch at 1 .

\section{Query Compiler}

Above, we discussed how to detect information defined in the relations in Fig. 3 through instrumentation primitives. However, we cannot afford first populating the relations and then answering queries. We rely on the query compiler to collect as little information as possible to answer queries. The compiler takes a query provided by the user, performs instrumented replay of the relevant hosts using Pin to collect the specific information needed to answer the query, and then performs the post-processing or aggregation necessary to present the results in the format requested by the user.

The compiler front end is a query parser. Because we support queries with a grammar structured like SQL, we leverage an existing SQL parser [3] to build an abstract syntax tree representing the query structure. Answering a single query then involves replaying precisely the desired subset of hosts with instrumentation that collects the information requested in the query. Determining which relations should be populated can be done by looking at the relations referenced in the query itself. The corresponding instrumentation primitives are enabled through command line options of Pin. A primitive can be easily attached to (or detached from) Pin by providing (removing) the corresponding instrumentation module name via the command line.

To avoid collecting too much information, it is necessary to control the elements or rows of the relations to be populated. That is, we never want to return results for the entire duration of any given host's execution, e.g., answering 
a query like "SELECT * FROM CF WHERE host=0", as it entails recording the entire control flow trace of a process. Hence, we only want to observe the relations at specific times or intervals. This is ensured through two different aspects of the system: WHERE clause filtering and join operations. First, let us consider simple queries over a single relation. In this case, the filters over which elements should be recorded are explicitly provided by the user in the form of the WHERE clause. Note that we require the WHERE clause to restrict at least one more field other than the host id when querying the State, DD, or $\mathbf{C D}$ relations because these relations reflect execution properties that may be viewed as continuous over the entire execution, yielding an intractable quantity of information.

When performing a join operation, or more specifically an inner join, results from a query over one relation are combined with results from another relation based on some common attributes. For example,

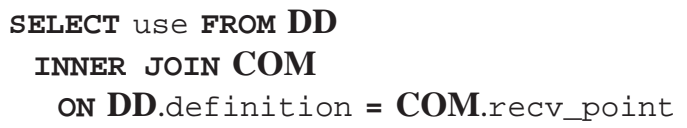

is a query that collects the execution points for all uses of variables defined at the reception of a message. Conceptually, it requires retrieving the DD and COM relations and then finding pairs of results that satisfy the join predicate as specified by on. In practice, however, implementing the query this way would be intractable, requiring not only the explicit capture of both already large relations, but also some filtering over their Cartesian product. To deal with this, we pipeline the data collection of the relations used within a join operation, such that the concrete results from one relation are explicitly used in the collection of another. For example, in the above join operation, the query over COM, returning all recv_points observed in the system, can be executed immediately. If that query is executed first, and the results from that query are used as the basis for restricting the query over DD, then finding a solution to the join can become practical because the number of communication events is expected to be tractable in practice.

\begin{tabular}{|r|l|}
\hline Precedence & Relations \\
\hline (High) 1 & Output \\
\hline 2 & Communication \\
\hline 3 & CD, DD, CF \\
\hline (Low) 4 & State \\
\hline
\end{tabular}

Table I: Relation precedence in joins.

To generalize the above observation, we thus roughly use the expected approximate size of the implicit relations to prioritize which information should be collected first. The priorities are given in Table I. For example, output is expected to be a relatively rare occurrence on any particular node in the system, so evaluating queries on the Output relation is often directly feasible. Thus, it has the highest pri- ority, and the results from an output query will be collected first in a join operation. Communication between nodes is not a rare event, but it does not happen at every instruction across all hosts, so COM has the next highest priority. The CD, DD, and CF relations all contain a bounded number elements at every instruction executed within the system, so they are all larger relations than COM and should only be evaluated afterward in order to winnow their results. Finally, State has a theoretically unbounded number of elements for every instruction executed on every host, so it clearly has the lowest priority when joining. In the event that the size of a result set still exceeds a maximum limit, we simply stop data collection and return the partial result set. The user can then refine the query further.

\section{REPLAY WITH INSTRUMENTATION}

This section describes the separation of logging from replay in Recon and the implementation of its replay system.

Low overhead is important during logging execution. In contrast, replay is often conducted during the debugging phase, in which overhead is not a major concern and more functionality is highly desirable. Hence, we separate Recon into two subsystems. One is the logging tool that is very lightweight for normal runs; the other is the replayer built on a powerful dynamic instrumentation infrastructure.

Achieving such a separation is, however, far from trivial. Ideally, we could have combined existing industrial strength tools such as Jockey to record an execution and Pin for dynamic instrumentation. Unfortunately, the nature and the complexity of the tools don't allow this to happen straightforwardly as we explain in the following.

Jockey runs in user space. When an application is run on Jockey, Jockey calls an initialization method before the application gains control, in which Jockey scans through the binary, including the libraries loaded by the application, looking for any syscall sites. Those syscalls are redirected to Jockey by overwriting the instructions at the syscall sites.

In contrast, the dynamic instrumentation engine Pin takes a binary. During execution, before executing any new (never instrumented) code regions, it calls a provided instrumenting function. This function instruments the given code regions and returns a new code region that contains both the original semantics and the instrumentation semantics that expose execution details. Pin executes the instrumented code instead of the original code. The instrumented code region is also copied to a new code space and thus it can be reused without calling the instrumentor again during the same execution. Note that such instrumentation is dynamic, meaning that it occurs during execution of the program.

Simple aggregation of the two tools can occur in two ways: one is Jockey+Pin+application, meaning that both Pin and the application run on top of Jockey; the other is Pin+Jockey+application. Alas, neither works. In the former case, Jockey gains control first and the subject program of 
Jockey is Pin instead of the application, which is itself the subject of Pin. Recall that Pin instruments the application on the fly during execution, and only the instrumented version gets executed. The instrumented version does not exist at the beginning, and hence its syscalls are not visible to Jockey. In fact, Jockey will search for syscalls in Pin and patch them. Thus, during Pin execution, all those syscalls will be undesirably redirected to Jockey.

Inversely, if Jockey and the application run on top of Pin, Jockey first intercepts all the syscalls of the application. The patches by Jockey and then the logging/replay code become part of the application. When the patched application executes inside Pin, Pin will try to instrument the patches and the Jockey code along with the application code. Such instrumentation is not only undesirable but often destructive because Jockey patches and code are at a very low level. Also, both tools reserve an overlapping virtual space region for their own purposes, which causes intricate conflicts. Finally, the threading models of the tools are incompatible. As a result, even after solving the compatibility problem, the integration would still fail on threading programs.

Our solution is to avoid using Jockey for replay. Instead, we implement the replay functionality inside Pin. The insight is that since Pin can potentially trap each instruction in the application, we can easily trap all the syscalls in the application. In particular, we implement an event log parser that can parse the logs generated by Jockey. Then we trap all the syscalls of the application inside Pin. Upon the execution of a syscall, we retrieve the corresponding event from the log through the parser. Note that Pin allows us to compose and attach multiple instrumentation modules exposing various aspects of application execution. We still benefit from the low logging overhead by using Jockey to generate logs.

\section{A. Handling Threading}

Jockey supports multithreaded applications using its emulation library called fakethread to allow maximal control over recording thread execution. Fakethread support is composed as a library that can be linked with threaded applications, providing the same interface as pthreads. It is completely inside user space and threads are opaque to the kernel, which is different from pthreads. Fakethread allows one thread to execute at a time. A thread is never preempted. Instead, it runs continuously until a blocking syscall, such as a blocking read or write, or until it fails to acquire a lock. In such cases, fakethread performs a (user space) context switch to the next ready thread. Jockey records all thread creation, join, termination, and context switch events.

In contrast, Pin's threading model is based on pthreads. It relies on the kernel to manage threads. In particular, the existence of a user thread is relayed to Pin by the kernel so that Pin can allocate space dedicated to the thread. Such space maintains thread local information related to analysis modules attached to Pin, e.g., a stack inside Pin for the thread in addition to the stack in user space. Pin does not interfere with thread scheduling. Context switches and synchronizations are managed by pthreads independently.

The differences between the two threading models make replaying Jockey logs inside Pin challenging. The problem stems from Pin not being aware of threads created through the user level fakethreads. As a result, thread specific space is not allocated inside Pin, leading to various problems.

\begin{tabular}{|c|c|}
\hline ion & \\
\hline 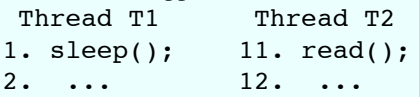 & $\begin{array}{l}\text { 21. intercept_sleep() } \\
\text { 22. ... } \\
\text { 23. ft_switch(); }\end{array}$ \\
\hline $\begin{array}{l}\text { Fakethread library } \\
\text { 31. ft_switch( ) \{ } \\
\text { 32. switch the user thread contexts; } \\
\text { 33. return; } \\
\text { \} }\end{array}$ & $\begin{array}{l}\text { 25. intercept_read () }\{ \\
\text { 26. readfrom } \log ; \\
\text { 27. ft_switch (); } \\
\text { \} }\end{array}$ \\
\hline
\end{tabular}

Figure 5: Example for fakethreads in Pin.

\section{B. Achieving Separation in Recon}

Consider an example in Fig. 5, in which fakethreads are used. The application has two threads, T1 and T2. Their respective calls to sleep() and read() lead to blocking syscalls. The Pin box shows the functions (inside Pin) that intercept the two syscalls, which in turn call the fakethread context switch function. The Fakethread box shows the context switch function inside the fakethread library. Upon a syscall from the application, Pin traps the call and dispatches the call to the right handler inside Pin, e.g., intercept_read() for the read() call in T2. The handler first reads the event from the log file. If the syscall is blocking, the fakethread context switch is called.

Fig. 6 shows a sample execution that executes $\mathbf{T} 1$ to the blocking syscall sleep() at line 1, then executes T2 to the blocking call read() at line 11, and finally switches back to T1, ending with a segmentation fault. The reason is the invisibility of fakethreads inside Pin. The stacks shown beneath the trace inside Fig. 6 explain the problem. Fig. 6(a) shows the stacks after line 32 and before the context switch. Upon the switch, the user stack is changed from T1 to T2. The problem lies in the trap of read () by Pin. Since Pin does not know that there are two threads, it uses the same internal stack such that the activation record of the intercept_read() invocation is pushed on top of that of intercept_sleep () (from T1), as shown in (b). As a result, in (c), when the fakethread scheduler switches back to $\mathbf{T 1}$, the user stack is correctly recovered but not the Pin stack. In particular, the return at line 33 would direct the control flow to intercept_read() while it should return to intercept_sleep ().

Our solution is to use real pthreads during replay so that Pin is aware of the user threads. In particular, we create pthreads during replay at places where the log indicates that fakethreads were created during the original run; pthread 


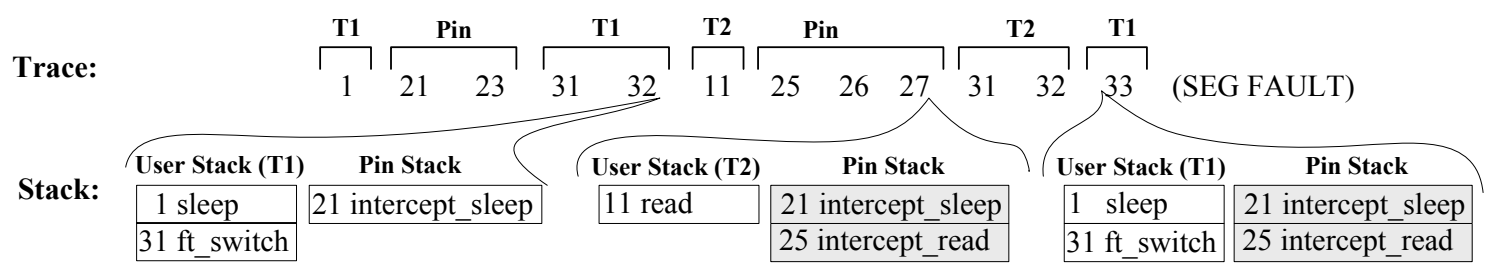

(a) After 32 in $\mathrm{T} 1$

(b) After 27 in Pin

(c) Before 33 in $\mathrm{T} 1$

Figure 6: Fakethreads are problematic. The highlighted Pin stacks cause the problem.

joins and exits also strictly follow the log. The challenge lies in forcing the pthread scheduler to respect the schedule specified by the fakethread log. More specifically, fakethreads are not preemptive, but pthreads' default scheduling policy is preemptive, e.g., a thread's time slice may expire, such that undesirable context switches may occur. Furthermore, the pthread scheduler is external to Pin; thus forcing a context switch between two specific threads is not straightforward.

We use a global pthread lock to enforce the recorded schedule. More specifically, a thread has to own the global lock to start or resume its execution. A thread yields the lock only when the fakethread log indicates a context switch. Hence, a pthread originated context switch during replay does not lead to the execution of a different thread if the $\log$ does not say so.

\section{EVALUATion}

To establish the practicality of Recon, we examined the runtime overhead it incurs for logging and replay. We also evaluated Recon on several real world bugs, including one unreported bug, from four distributed applications.

\section{A. Efficiency}

We first examined the runtime overhead incurred for both the logging that occurs in original runs and for the instrumented replays in the debugging phase. Overhead was measured on a set of four distributed programs including BerkeleyDB, i3/Chord, Pastry, and RandTree. BerkeleyDB is a popular open-source database engine that provides data replication capability to enable a group of processes to service the workload. The Pastry and RandTree implementations are those from the Mace infrastructure [12]. All tests were performed on an Intel dual core $1.66 \mathrm{GHz}$ machine with $3 \mathrm{~GB}$ memory. Individual nodes in the network were executed in independent virtual machines, each running Linux 2.6.11.We also measured the logging overhead of BerkeleyDB on a real distributed environment, Amazon EC2. Each node had $2.66 \mathrm{GHz} \mathrm{CPU}$ with $1.7 \mathrm{~GB}$ memory.

Let us first consider the runtime overhead during logging and uninstrumented replay (on Pin), as presented in Fig. 7. Here we deploy 4 nodes for all benchmarks. We use the insertion of $10,000 \mathrm{key} / \mathrm{value}$ pairs as the input to BerkeleyDB. For I3/Chord, we use the test suite provided by I3. We use the appmacedon tool, included in the Mace release,

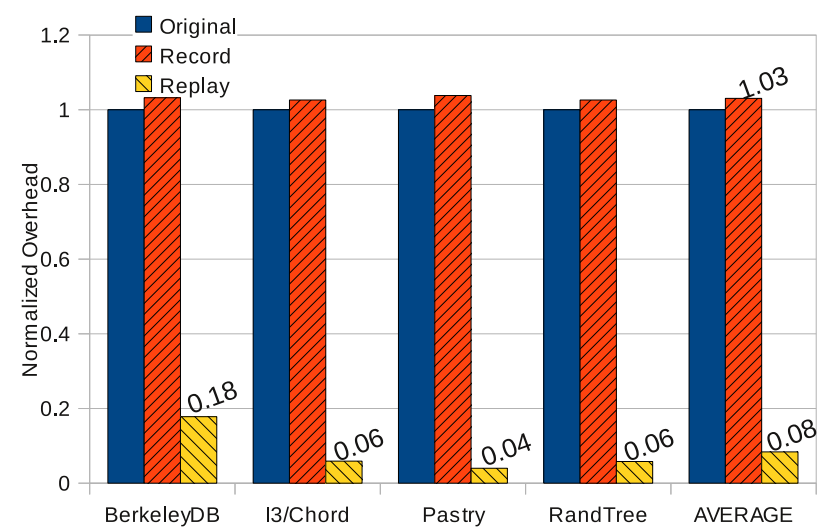

Figure 7: Logging/replay overhead.

as a driver for multicast and randomly generated streaming data to measure overhead of RandTree and Pastry. All times are normalized against the original execution time. Original marks the unmodified runtime of the original program. Record denotes the logging time. It was measured from the starting time of the first action of the system to the finishing time of the last action. Replay denotes the most significant overhead for replaying a single node in the system. Replay time is normalized against the original time for that same node. Note that our system allows multiple node replay, controlled by the query compiler.

Observe first that the logging overhead is both consistent and tolerable. The maximum overhead is only $4 \%$, for Pastry, and all other cases have $3 \%$ overhead, which is tolerable for realistic runs. For replay, the overhead results appear surprising at first. Indeed, the time taken for replay is significantly less than the original run and only $8 \%$ of the original run on average. This results from the time saved by emulating all system calls during replay. When no waiting time is incurred during replayed system calls, the overall execution is, in fact, much faster than the original.

We also vary the size of our deployment to observe overhead changes for BerkeleyDB. We use the same input and vary the number of nodes from 1 to 8 . We executed the same set of experiments on both the local machine and Amazon EC2 cluster. Note that the same set of data is replicated to all nodes in the system. The results are presented in Table II. Observe that the logging overhead is high for one node but less for other settings. The reason is that the communication delay masks some overhead when 


\begin{tabular}{|c|c|c|c|c|c|}
\hline \multirow{2}{*}{ \# of nodes } & \multicolumn{2}{|c|}{ exec. time (s) } & \multicolumn{2}{c|}{ logging overhead } & \multirow{2}{*}{$\log$ size } \\
\cline { 2 - 5 } & Local & EC2 & Local & EC2 & \\
\hline 1 & 180.5 & 25.58 & $7.19 \%$ & $2.88 \%$ & $6.79 \mathrm{MB}$ \\
\hline 2 & 414.4 & 396.33 & $3.14 \%$ & $0.54 \%$ & $9.13 \mathrm{MB}$ \\
\hline 4 & 512.0 & 212.74 & $3.19 \%$ & $1.11 \%$ & $12.28 \mathrm{MB}$ \\
\hline 8 & 594.7 & 313.4 & $3.04 \%$ & $1.05 \%$ & $17.07 \mathrm{MB}$ \\
\hline
\end{tabular}

Table II: Logging costs vs. \# Nodes for BerkeleyDB.

multiple nodes are involved. The execution time with 2 nodes is a lot slower than the 1 node execution because the data has to be replicated to the second node. When the node count goes higher, the overhead does not increase as substantially because the replication is done through broadcasting. In the EC2 cluster, execution time with 2 nodes is slower than the 4 node execution because the master node must wait until receiving at least one acknowledgment message from clients for each input data. When we have 2 nodes, one master and one client, the only client needs to store all replicas and sends ack messages to the master, but in the 4 node case, any available client can send ack to the master. When we have 8 nodes, the master node needs at least 3 ack messages from clients, so the execution time is slower than with 4 nodes but still faster than the 2 node case. Local machine execution does not show this symptom because increasing the number of nodes causes CPU and memory congestion. The log file sizes are also presented in the last column.

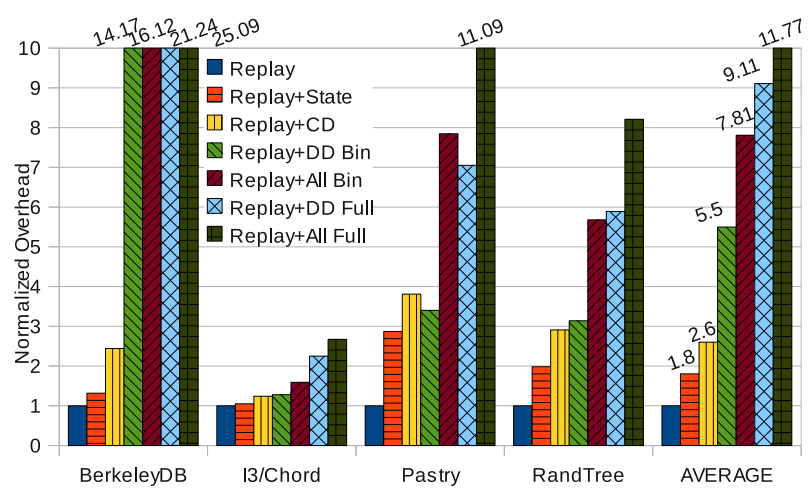

Figure 8: Instrumentation overhead.

The instrumentation overhead for collecting several different execution artifacts is presented in Fig. 8. Once again, all runtimes are normalized, this time against the replay time for each program. Replay presents the base replay time, i.e., replay inside Pin without instrumentation. Replay+State gives the time necessary to locate a particular point in the replay of a node such that the requested state can be extracted with the help of dwarfdump. Note that populating the Output and Communication relations demands the same online instrumentation and hence the same overhead applies to those primitives as well. Replay $+C D$ gives the time necessary for detecting the control dependence for each execution point within a node. Replay $+D D$ Bin presents the time for detecting the data dependence for each execution point in the application binary while Replay $+D D$ Full covers both the application binary and libraries. We separate these two options because instrumenting libraries may not be necessary for all programs. BerkeleyDB builds some of its own libraries as well, so those are included in its Bin results. Replay + All Bin presents the overhead of all instrumentation primitives for the application binary while Replay+All Full does so for both the binary and the libraries.

In all cases, tracking the execution position to collect state information takes less than 3 times the replay runtime, and in most cases, it takes less than $2 \times$ as long to execute, $1.8 \times$ on average. Tracking control dependence alone usually has less than $3 \times$ overhead with respect to the base replay runtime, $2.6 \times$ on average. For most benchmarks, data dependence is the most expensive execution artifact to detect, with full data dependence detection, including through library calls, taking an average of $9.11 \times$ to execute. Note that the overhead from BerkeleyDB is the dominant contributor to this average. The BerkeleyDB tests involve significantly more data than those for the other programs, resulting in a higher number of data dependencies being detected. Sometimes, it may be beneficial to exclude data dependencies that occur inside external library calls. When we do this, tracking data dependence can be done with $5.5 \times$ the base runtime on average. Having all primitives activated incurs more slowdown. Overall, considering that replay is much faster than the original run $(0.08 \times)$, the instrumented runs are even faster or comparable to the original runs. Such overhead is acceptable in the debugging phase.

\section{B. Effectiveness}

We tested our tool with eight bugs from four distributed applications. One of them (RandTree \#1) is an unreported bug and we can easily generate queries to find a root cause. We will discuss details in the Case Study \#2. Fig. 9 presents the total number of queries generated for debugging, and also the frequency with which they involved each relation from Fig. 3. The right-most column represents the number of nodes we needed to replay in order to find the cause of each bug. From the table, we can observe that all these real bugs can be resolved in a few queries with 14 being the maximum, showing the effectiveness of our technique. Queries at different levels, from variable state to node interactions, are needed, and most of these bugs require reasoning across node boundaries, illustrating the necessity of a unified debugging view. Next, we will present a few case studies.

Case Study \#1: The first case is a failure of a leader election scheme in BerkeleyDB 4.7.25 that supports the single master/multiple clients model. This bug was originally analyzed in [32].

When a master crashes, all remaining nodes in the system automatically start a new round of leader election. However, in the case of this bug, the system permanently fails to elect a new master because all nodes in the system believe 


\begin{tabular}{|c|c|c|c|c|c|c|c|c|c|}
\hline Bug & Summary & Total Queries & STATE & CD & DD & OUTPUT & $\mathbf{C F}$ & COM & \# of nodes \\
\hline$\overline{\mathrm{DB} \# 1}$ & Permanent election failure [32] & $\overline{13}$ & $\overline{11}$ & 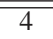 & 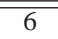 & $\overline{11}$ & $\overline{0}$ & $\overline{2}$ & $\overline{2}$ \\
\hline DB\# 2 & Master node panic [32] & 14 & 1 & 3 & 8 & 1 & 0 & 1 & 2 \\
\hline Chord\#1 & Packet handling failure [9] & 7 & 3 & 2 & 3 & 1 & 0 & 0 & 1 \\
\hline Chord\#2 & Inconsistent ring [10] & 7 & 0 & 2 & 1 & 1 & 2 & 2 & 2 \\
\hline RandTree\# 1 & Permanent join fail & 9 & 0 & 2 & 4 & 1 & 0 & 3 & 3 \\
\hline RandTree\#2 & Disjoint tree [10] & 7 & 0 & 3 & 2 & 1 & 1 & 1 & 2 \\
\hline RandTree\#3 & Fail to find peer [13] & 5 & 1 & 1 & 3 & 1 & 0 & 1 & 2 \\
\hline Pastry\#1 & Buffer overflow [13] & 5 & 1 & 2 & 1 & 1 & 0 & 1 & 2 \\
\hline
\end{tabular}

Figure 9: Analyzed bugs with the number of queries used and relation usage distributions.

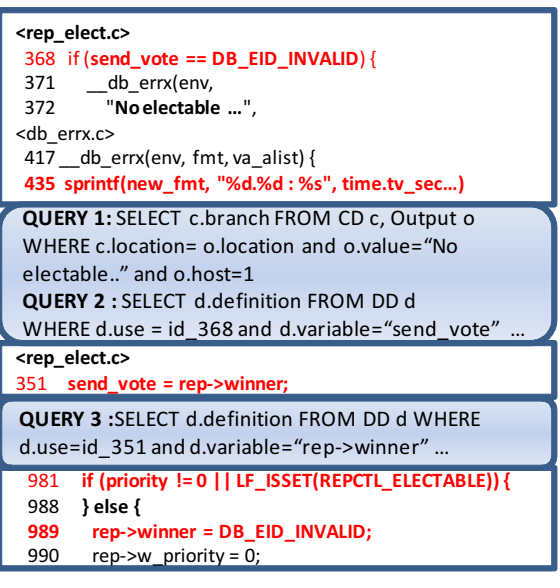

\begin{tabular}{|c|}
\hline $\begin{array}{l}\text { QUERY 4: SELECT c.branch FROM CD c WHERE } \\
\text { c.location=id_989 and c.host=1 } \\
\text { QUERY 5: SELECT s.value FROM State s WHERE } \\
\text { s.location=id_981 and s.variable="priority" }\end{array}$ \\
\hline $\begin{array}{l}\text { QUERY } 6 \text { : SELECT d.definition FROM DD d WHERE } \\
\text { d.use=id_ } 981 \text { and d.variable="priority" ... }\end{array}$ \\
\hline $\begin{array}{l}252 \text { if (rep->flags \& (REP_F_READY_API | } \\
\text { REP_F_READY_OP | REP_F_RECOVER_LOG )) \{ } \\
257 \text { priority = 0; }\end{array}$ \\
\hline $\begin{array}{l}\text { QUERY 7: SELECT c.branch FROM CD c WHERE } \\
\text { c.location=id_ } 257 \text { and c.host=1 } \\
\text { QUERY } 8 \text { : SELECT d.definition FROM DD d WHERE } \\
\text { d.use=id_ } 252 \text { and d.variable="rep->flags" ... }\end{array}$ \\
\hline $\begin{array}{l}<\text { rep_record.c > } \\
476 \text { switch (rp->rectype) }\{ \\
873 \text { case REP_UPDATE: } \\
878 \text { ret }=\text { _rep_update_setup(env, eid, rp, rec); } \\
<\text { rep_backup.c > } \\
\text { rep_update_setup(..) \{ } \\
2097 \quad \text { F_SET(rep, REP_F_RECOVER_LOG); }\end{array}$ \\
\hline
\end{tabular}

QUERY 9 : SELECT c.branch FROM CD c WHERE
c.location=id_2097 and c.host=1
QUERY $10:$ SELECT d.definition FROM DD d WHERE
d.use=id_476 and d.host=1
<rep_auto.c>
129 DB_NTOHL_COPYIN(env, argp->rectype, bp);
QUERY 11 : SELECT d.definition FROM DD d WHERE
d.use=id_129 and DD.variable=“bp” and DD.host=1
<repmgr_posix.c>
521 ... readv(fd, iovec, buf_count)...
QUERY 12 : SELECT COM.sender, COM.send_point
FROM COM WHERE COM.receiver=1 and
COM.recv_point=id_521
Sender is NODE 0
2112 (void)_rep_send_message(env, eid, ... )
<repmgr_posix.c>
503 ... writev(fd, iovec, buf_count)...
QUERY 13:SELECT COM.receiver FROM COM WHERE
COM.sender=0 and COM.send_point > id_521

Figure 10: Case study 1 : BerkeleyDB - failure in election.

they cannot be the master. Suppose node 0 is the original master node and is synchronizing data with all other client nodes, nodes 1 and 2 . The steps of synchronization are as follows. First, when node 0 receives modified data, it sends a REP_PAGE message with the modified data to all clients. All clients who receive this message change their state to LOG_RECOVERY by setting a flag to REP_F_RECOVERY_LOG, meaning they expect the corresponding log update in the next step. When this flag is set, the node cannot be a master. The clients send a REP_LOG_REQ message to the master node, requesting the updated $\log$. In the next step, when node 0 receives the $R E P \_L O G \_R E Q$ message, it sends a REP_LOG message with the updated log records. When clients receive the REP_LOG message from the master, they restore to the normal state. The bug happens when node 0 is down after it sends a REP_PAGE message but before sending the REP_LOG message. When the master is down, all clients start election but permanently fail to elect a new master. The symptom is an error message on all client nodes - "No electable site found : ..."

We generated 13 queries. Figure 10 presents the code snippets related to the failure appended with the corresponding queries. We started debugging with node 1 , which is one of the client nodes that printed the error message. From QUERY1 (Q1), we can obtain the execution point where the message was printed, line 435 , and we can also reach the branch point controlling the execution there, which is line 368. From the first query, we find that this error happens be- cause variable send_vote has the value DB_EID_INVALID. Now we want to know where the value of send_vote was defined. In Q2, we query the data dependence of send_vote at line 368 . Here, we use id_368 to denote the execution point of line 368 for readability. Q2 returns the execution point of line 351, and now we need to know where the variable $r e p \rightarrow$ winner was defined. From Q3, we reach line 989, where $r e p \rightarrow$ winner was given the constant value DB_EID_INVALID. Now we need to obtain the control dependence of the execution point at line 989. Q4 shows that line 981 controls line 989. In order to decide which condition caused the wrong branch outcome, we query the value of priority at 981 (Q5), which reveals that it has an undesirable value of 0. Through Q6 and Q7, we reach line 252, the branch controlling the definition of priority to 0 at line 257 .

From queries Q8-11, we can reach the line 521, where the message was received from another node. At this point, Q12 reveals that that node 1 has received the message from node 0 at line 503. Node 0 was supposed to send REP_LOG after the execution of line 503 (sending REP_PAGE). However, according to Q13, whose result is an empty set, node 0 did not send any further message to node 1. From these combined queries, we thus discover the reason for the election failure.

Case Study \#2: The second case study is for a previously unreported bug in RandTree. This bug exhibits as a permanent failure when a node joins the tree. In the experiment, we 

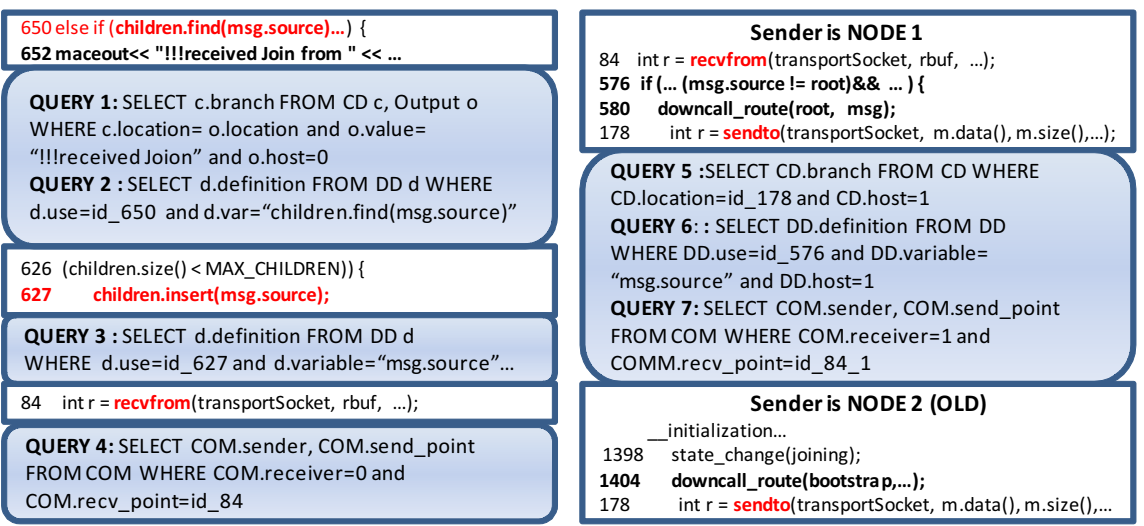

\begin{tabular}{|c|}
\hline $\begin{array}{l}\text { QUERY } 8 \text { : SELECT DD.definition FROM DD } \\
\text { WHERE DD.use=id_ } 650 \text { and DD.variable="msg.source" } \\
\text { and DD.host=0 }\end{array}$ \\
\hline $\begin{array}{l}84 \text { int } r=\text { recvfrom (transportSocket, rbuf, } \\
\text { MAX_MESSAGE_SIZE, 0, (struct sockaddr*)\&sa, \&salen); }\end{array}$ \\
\hline $\begin{array}{l}\text { QUERY 9: SELECT COM.sender, COM.send_point } \\
\text { FROM COMM WHERE COMM.receiver }=0 \text { and } \\
\text { COMM.recv_point=id_84_2 }\end{array}$ \\
\hline 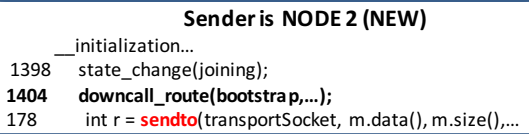 \\
\hline
\end{tabular}

Figure 11: Case study 2: RandTree - permanent failures to join tree.

have two nodes in the tree, nodes 0 and 1 , in the initial phase. Later, node 2 starts and sends a join message to node 1 . The message is relayed to the root node 0 . Node 0 sends a join reply message to node 2 and updates its own child list, which now includes node 2 , but node 2 resets right after sending the join message. After node 2 is reset, it ignores the join reply message from node 0 because it is in the initialization phase. After node 2 finishes initialization, it sends a join message to node 0 . Node 0 ignores that message because node 2 is already its child. Node 2 keeps sending join messages to node 0 , but all of them are ignored and thus node 2 cannot join the tree. When node 0 ignores the join message from node 2 , it prints the error message "!!! received Join from $x x$, already child."

We used 9 queries to debug this failure, all presented in Fig. 11. We started from the error message. After Q1, we reach line 650, meaning node 2 is found in the child list. From this point, we seek to know why node 2 is already in the list. Using Q2, we find that node 2 is added at line 627 , as the result of a message received at line 84 (Q3). Q4 discloses that the message was sent from node 1, as the result of a message received at line 84 in node 1 (Q5-6). Q7 identifies that the message was from the previous process of node 2 expressing its intention of joining the tree. Node 1 simply relayed the join message. Then, we go back to line 650 and try to understand why msg. source has the value of node 2. Through Q8 and Q9, we know that this join is from the new node 2 process.

The full queries for the remaining bugs in Fig. 9 are omitted due to space restrictions.

\section{RELATED WORK}

Debugging pervasive systems is becoming increasingly important. D3S [15] and WiDS [14] are projects that aim to detect runtime errors in distributed systems. They are based on runtime property checking at the event level. These systems assume the user knows exactly what properties to check. In contrast, our system provides an interface that exposes artifacts at various levels, allowing the user to examine them regardless of foresight, starting from the observed symptoms. Another thread of work for debugging distributed systems uses model checking [19], [31]. MaceMC [12] is an explicit state model checker that checks liveness properties. In general, model checking also requires the user to have prior knowledge about the property to check and generally scales poorly. CrystalBall [30] is a tool that checks predefined properties on the fly.

In [25], Singh et al. propose a declarative language based debugging interface that is similar to our SQL interface. However, their system is specialized to their own declarative pervasive system programming language [16]. Our system is much more general, working on arbitrary binaries. Ptql [11] and pql [18] propose query languages for single process execution. These techniques are not combined with replay and their schema design does not focus on debugging. Magpie [5], Pinpoint [6], and Pip [23] are projects based on log mining. In other words, they try to identify problems by looking at event logs. These techniques are quite effective in debugging performance problems, but less so for faults. In [29], Xin et al. present a technique to analyze distributed systems by building task graphs from event log files. Pothier et al. [22] present a portable Trace-Oriented Debugger for Java which uses efficient instrumentation techniques for event generation and a scalable storage system for completeness and efficient querying. All attributes of all events are logged at all times which is considered feasible in the targeted centralized setting targeted and with the assumption of a wireline connection to a dedicated scalable backend. Logging and replay [7], [9], [26], [21] is an important strategy for pervasive debugging. Existing work focuses on single node replay, which is insufficient for pervasive debugging. Friday's replay system [10] supports replay with a GDB-like interface, but it cannot handle fine-grained instrumentation.

\section{CONCLUSION}

We have presented Recon, a system for debugging distributed systems based on a novel architecture, providing a consistent view of salient system properties. This view 
exposes properties via a relational framework that can be queried with a simple language based on SQL. Information collection is performed on demand to answer the queries, using filtering and prioritization to avoid collecting data unnecessary to formulating the answer.

We qualitatively evaluated Recon on several bugs in popular distributed programs. Furthermore, we have evaluated our design that separates a logging infrastructure from heavyweight analyses during replay, showing that it allows Recon to be used to record realistic runs with acceptable overhead $(3 \%)$ and debug the runs later by replaying them.

\section{ACKNOWLEDGMENT}

We would like to thank the anonymous reviewers for their insightful comments. This research is supported in part by the National Science Foundation (NSF) under grants 0845870 and 0834529 . Any opinions, findings, and conclusions or recommendations in this paper are those of the authors and do not necessarily reflect the views of NSF.

\section{REFERENCES}

[1] dwarfdump, http://reality.sgiweb.org/davea/dwarf.html.

[2] Macedon, http://www.macesystems.org/macedon.

[3] python-sqlparse, http://code.google.com/p/python-sqlparse.

[4] M. Balazinska, H. Balakrishnan, and D. Karger, "Ins/twine: a scalable peer-to-peer architecture for intentional resource discovery," in Pervasive, 2002.

[5] P. Barham, R. Isaacs, R. Mortier, and D. Narayanan, "Magpie: online modelling and performance-aware systems," in HotOS, 2003.

[6] M. Y. Chen, E. Kiciman, E. Fratkin, A. Fox, and E. A. Brewer, "Pinpoint: problem determination in large, dynamic internet services," in DSN, 2002.

[7] G. W. Dunlap, S. T. King, S. Cinar, M. A. Basrai, and P. M. Chen, "Revirt: enabling intrusion analysis through virtualmachine logging and replay," in OSDI, 2002.

[8] J. Ferrante, K. J. Ottenstein, and J. D. Warren, "The program dependence graph and its use in optimization," $A C M$ TOPLAS, vol. 9, no. 3, 1987.

[9] D. Geels, G. Altekar, S. Shenker, and I. Stoica, "Replay debugging for distributed applications," in USENIX, 2006.

[10] D. Geels, G. Altekar, P. Maniatis, T. Roscoe, and I. Stoica, "Friday: global comprehension for distributed replay," in NSDI, 2007.

[11] S. Goldsmith, R. O'Callahan, and A. Aiken, "Relational queries over program traces," in OOPSLA, 2005.

[12] C. E. Killian, J. W. Anderson, R. Jhala, and A. Vahdat, "Life, death, and the critical transition: finding liveness bugs in systems code," in NSDI, 2007.

[13] C. E. Killian, "Systems and language support for building correct, high performance distributed systems," in Ph.D. dissertation. University of California, San Diego, 2008.
[14] X. Liu, W. Lin, A. Pan, and Z. Zhang, "WiDS checker: combating bugs in distributed systems," in NSDI, 2007.

[15] X. Liu, Z. Guo, X. Wang, F. Chen, X. Lian, J. Tang, M. Wu, M. Kaashoek, and Z. Zhang, "D3S: Debugging deployed distributed systems," in NSDI, 2008.

[16] B. T. Loo, T. Condie, J. M. Hellerstein, P. Maniatis, T. Roscoe, and I. Stoica, "Implementing declarative overlays," in SOSP, 2005.

[17] C. Luk, R. Cohn, R. Muth, H. Patil, A. Klauser, G. Lowney, S. Wallace, V. J. Reddi, and K. Hazelwood, "Pin: building customized program analysis tools with dynamic instrumentation," in PLDI, 2005.

[18] M. C. Martin, V. B. Livshits, and M. S. Lam, "Finding application errors and security flaws using PQL: a program query language," in OOPSLA, 2005.

[19] M. Musuvathi and D. R. Engler, "Model checking large network protocol implementations," in NSDI, 2004.

[20] N. Nethercote and J. Seward, "Valgrind: A framework for heavyweight dynamic binary instrumentation," in PLDI, 2007.

[21] S. Park, W. Xiong, Z. Yin, R. Kaushik, K. Lee, S. Lu, and Y. Zhou. "Pres: probabilistic replay with execution sketching on multiprocessors," in SOSP, 2009.

[22] G. Pothier, E. Tanter, and J. Piquer, "Scalable omniscient debugging," in OOPSLA, 2007.

[23] P. Reynolds, C. E. Killian, J. L. Wiener, J. C. Mogul, M. A. Shah, and A. Vahdat, "Pip: detecting the unexpected in distributed systems," in NSDI, 2006.

[24] Y. Saito, "Jockey: a user-space library for record-replay debugging," in AADEBUG, 2005.

[25] A. Singh, P. Maniatis, T. Roscoe, and P. Druschel, "Using queries for distributed monitoring and forensics," in EuroSys, 2006.

[26] S. M. Srinivasan, S. Kandula, C. R. Andrews, and Y. Zhou, "Flashback: a lightweight extension for rollback and deterministic replay for software debugging," in ATEC, 2004.

[27] I. Stoica, R. Morris, D. Karger, M. Kaashoek, and H. Balakrishnan, "Chord: a scalable peer-to-peer lookup service for internet applications," in SIGCOMM, 2001.

[28] B. Xin and X. Zhang, "Efficient online detection of dynamic control dependence," in ISSTA, 2007.

[29] B. Xin, P. Eugster, X. Zhang, and J. Yang, "Lightweight task graph inference for distributed applications," in SRDS, 2010.

[30] M. Yabandeh, N. Knezevic, D. Kostic, and V. Kuncak, "Crystalball: predicting and preventing inconsistencies in deployed distributed systems," in NSDI, 2009.

[31] J. Yang, P. Twohey, D. R. Engler, and M. Musuvathi, "Using model checking to find serious file system errors," $A C M$ TOCS, vol. 24, no. 4, 2006.

[32] J. Yang, T. Chen, M. Wu, Z. Xu, X. Liu, H. Lin, M. Yang, F. Long, L. Zhang, and L. Zhou, "MODIST: transparent model checking of unmodified distributed systems," in NSDI, 2009. 